\title{
Diurnal intraocular pressure profiles and progression of chronic open-angle glaucoma
}

${ }^{1}$ Department of Ophthalmology, Faculty of Clinical Medicine Mannheim, University of Heidelberg, Mannheim, Germany

${ }^{2}$ Department of Ophthalmology, University Erlangen-Nürnberg, Erlangen, Germany

${ }^{3}$ Department of Biometry and Clinical Epidemiology, Campus Benjamin Franklin, Berlin, Germany

Correspondence: JB Jonas, Department of Ophthalmic, Heidelberg University, Universitäts-Augenklinik, Theodor-Kutzer-Ufer 1-3, Mannheim 68167, Germany Tel.: + 49621383 2652;

Fax: + 496213833803 .

E-mail: Jost.Jonas@

augen.ma.uni-heidelberg.de

Received: 9 November 2005 Accepted in revised form: 7 March 2006

Published online: 7 April 2006

Proprietary interest: none

\begin{abstract}
Purpose To evaluate whether the amplitude of day-and-night intraocular pressure (IOP) profiles influences the rate of progression of chronic open-angle glaucoma.

Methods The hospital-based clinical observational study included day-and-night profiles of IOP measurements performed on 458 patients (855 eyes) with chronic open-angle glaucoma or ocular hypertension. The 24-h pressure profiles obtained by Goldmann applanation tonometry contained measurements at 0700, noon, 1700, 2100, and midnight.

Results In the whole study population, IOP amplitude was significantly $(P<0.001)$ and positively associated with the mean $(r=0.26)$, minimal $(r=-0.23)$ and maximal $(r=0.59)$ IOP values. Taking the whole study population, glaucoma progression was not associated with the IOP amplitude $(P=0.09)$. After adjustment for age, neuroretinal rim area and the other IOP measurements, age $(P<0.001)$ and neuroretinal rim area $(P=0.05)$ remained as significant predictive factors in the selected Cox model. In the normal-pressure glaucoma group ( $n=174$ eyes), progression was significantly positive associated with the minimal IOP value $(P<0.001)$, the mean of the IOP values $(P=0.024)$, and, less significantly $(P=0.037)$ and negatively, with the pressure profile amplitudes. In the high-pressure glaucoma group ( $n=681$ eyes), rate of glaucoma progression was not associated with the IOP amplitude $(P=0.734)$ or the other IOP parameters.

Conclusions Taking into account the highly significant associations between the IOP amplitude and the mean, minimal and maximal IOP values suggests that it is the IOP itself, and less the pressure amplitude,
\end{abstract}

JB Jonas ${ }^{1,2}$, WM Budde ${ }^{1,2}$, A Stroux ${ }^{3}$, IM Oberacher-Velten ${ }^{1,2}$ and A Jünemann which has the main influence on the rate of the glaucoma progression.

Eye (2007) 21, 948-951; doi:10.1038/sj.eye.6702351; published online 7 April 2006

Keywords: intraocular pressure; glaucoma; intraocular pressure profiles; glaucoma progression

Introduction

Recent studies have revealed that the rate of progression of chronic open-angle glaucoma is associated with several parameters such as stage of the disease, occurrence of optic disc haemorrhages and level of intraocular pressure (IOP). ${ }^{1-3}$ The purpose of the present study was to evaluate whether the amplitude of day-andnight IOP measurements ${ }^{4-12}$ is an additional factor associated with the progression of glaucoma.

\section{Methods}

The clinical observational study included dayand-night IOP profiles measured on 855 eyes of 458 Caucasian subjects with chronic open-angle glaucoma or ocular hypertension (Table 1). Mean follow-up time was $55.6 \pm 35.1$ months (median 51.5 months; range 5.4-124.9 months). All subjects and patients included in the study had an open anterior chamber angle, and a best corrected visual acuity of $20 / 25$ or better. Exclusion criteria were all eye diseases other than glaucoma, diabetes mellitus, and corneal diseases. Informed consent was obtained from each subject before enrolment. The patients were part of a prospective glaucoma study (Erlangen Glaucoma Register). Institutional Review Board/Ethics Committee approval had been obtained at the start of the study. 
Table 1 Demographic description of the study groups $($ mean \pm SD)

\begin{tabular}{lccc}
\hline & $\begin{array}{c}\text { Whole study } \\
\text { population }\end{array}$ & $\begin{array}{c}\text { Normal- } \\
\text { pressure } \\
\text { glaucoma group }\end{array}$ & $\begin{array}{c}\text { High-pressure } \\
\text { glaucoma } \\
\text { group }\end{array}$ \\
\hline$n$ & 855 & 174 & 681 \\
Females/males & $426 / 429$ & $125 / 49$ & $301 / 380$ \\
Age (years) & $49.1 \pm 12.4$ & $55.2 \pm 10.7$ & $47.5 \pm 12.3$ \\
Median & 51.2 & 49.2 & 58.0 \\
\hline
\end{tabular}

The whole study group was divided into eyes with primary open-angle glaucoma, ocular hypertensive eyes and eyes with normal-tension glaucoma. In the eyes affected by primary open-angle glaucoma, no obvious reason for the elevated IOP higher than $21 \mathrm{mmHg}$ could be detected. Criteria for the diagnosis of normal-pressure glaucoma were maximal IOP readings equal to or less than $21 \mathrm{mmHg}$ in at least two 24 -h pressure profiles obtained by slit lamp applanation tonometry and containing measurements at 1700, 2100, midnight, 0700 and noon. Ocular hypertensive eyes had IOP measurements higher than $21 \mathrm{mmHg}$ without visual field defects and without glaucomatous abnormalities of the optic nerve head. Preperimetric glaucoma was defined by glaucomatous abnormalities of the optic nerve head and normal white-on-white visual fields. Perimetric glaucoma was defined by glaucomatous abnormalities of the optic nerve head and glaucomatous visual field defects. A glaucomatous visual field defect was defined as an Octopus G1 field with (a) at least three adjacent test points having a deviation of equal to or greater than $5 \mathrm{~dB}$ and with one test point with a deviation greater than $10 \mathrm{~dB}$ lower, (b) at least two adjacent test points with a deviation $\geq 10 \mathrm{~dB}$, (c) at least three adjacent test points with a deviation $\geq 5 \mathrm{~dB}$ abutting the nasal horizontal meridian, or (d) a mean visual field defect of more than $2 \mathrm{~dB}$. Rate of false positive answers and rate of false negative answers had to be $\leq 15 \%$. To define the baseline of the visual field examinations, two visual field tests performed prior to inclusion into the study were taken. Glaucomatous changes of the optic nerve head included an unusually small neuroretinal rim area in relation to the optic disc size, an abnormal shape of the neuroretinal rim, or cup to disc diameter ratios being vertically higher than horizontally, and localized or diffuse retinal nerve fibre layer defects. ${ }^{13}$ Since all glaucoma eyes examined in the study had to show glaucomatous abnormalities of the optic disc, the inclusion of normotensive eyes with non-glaucomatous optic nerve damage into the normal-tension glaucoma subgroup was not likely.

The patients were on routine ophthalmic care including topical application of antiglaucomatous medication. There was no major difference in the type of treatment between the various glaucoma subgroups with most of the eyes receiving topical betablockers twice daily and a prostaglandin analogue in the evening. IOP was measured by slit lamp Goldmann applanation tonometry. For all eyes included in the study, at least one day-and-night IOP profile was performed containing measurements at 1700, 2100, midnight, 0700, and noon. For the measurements at night, the patients either had stayed awake till midnight, or they were woken up and waited at least $15 \mathrm{~min}$ in upright position before the measurements were taken.

Progression of glaucomatous visual field loss was defined by point wise regression analysis for each of the 59 locations in the visual field. Point wise progression was assumed, if a difference of larger than $1 \mathrm{~dB}$ per year was observed for the local defect. A point wise improvement (learning effect or random variation) was assumed if a difference smaller than $-1 \mathrm{~dB}$ was observed. An eye was classified as progressive, if the number of locations with progression was significantly higher compared to the number of locations with improvement (binomial test, $P=0.05$ two-sided). ${ }^{14}$ For each eye, the first follow-up measurement with progression as defined above entered the analysis.

Descriptive statistics include absolute and relative frequencies for categorical, and mean, standard deviation, median, and range for numeric variables. For confirmatory analyses concerning correlations between IOP fluctuation and mean, maximum, and minimum IOP, Spearman's correlation coefficients were used and twosided $P$-values are presented. Progression was treated as a time to event variable. Actual time to progression in contrast to observed time to progression was estimated by the mid point of the interval between two visits. ${ }^{15}$ For univariate survival analysis, the log-rank test according to the sample terciles was used and Kaplan Meier curves for the IOP amplitudes are presented (Figures 1 and 2). Cox proportional hazard regression analysis with forward selection was applied in order to identify independently predictive factors for progression. Dependency of left and right eyes from the same subject was taken into account conservatively: $\chi^{2}$ values were multiplied by the factor 'number of patients divided by number of eyes'. Thus significance tests were rather performed with respect to the number of patients instead of the number of eyes. Only two-sided $P$-values are presented. Statistical analysis was performed by using a commercially available statistical software package (SPSS for Windows, version 11.5, SPSS, Chicago, IL).'

\section{Results}

Out of the 855 eyes, 163 (19.1\%) eyes showed progression of glaucoma. Dividing the group into a normal-pressure 


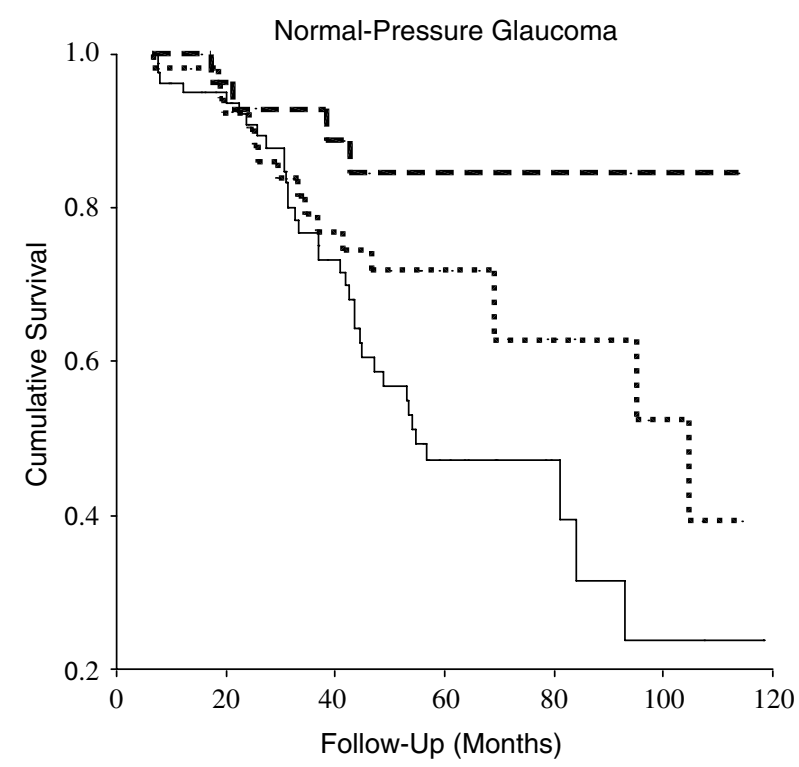

Figure 1 Kaplan-Meyer curve showing the rate of progression in relation of the height of the amplitude of IOP profiles in eyes with normal-pressure glaucoma $(n=174$ eyes).

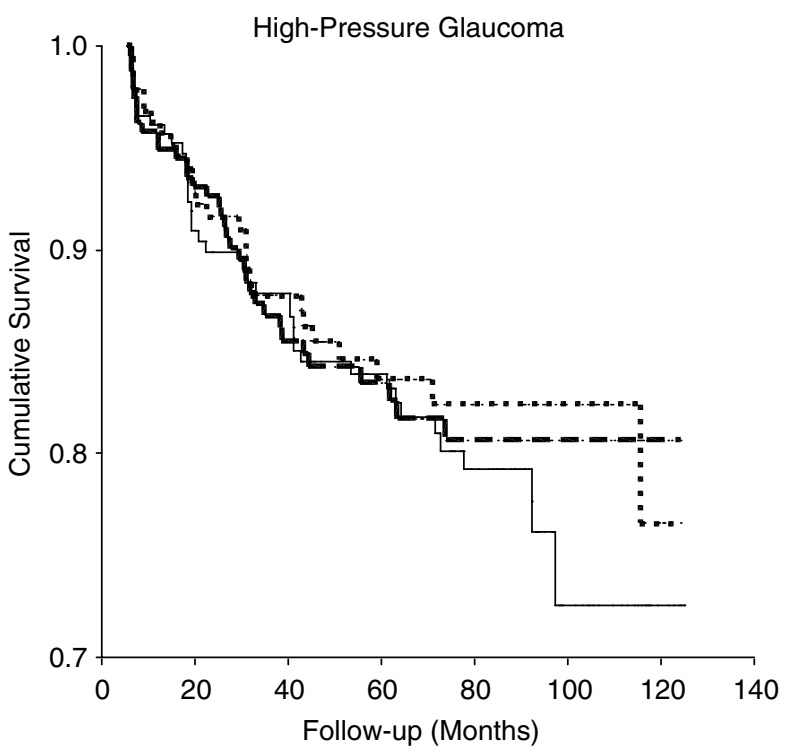

Figure 2 Kaplan-Meyer curve showing the rate of progression in relation of the height of the amplitude of IOP profiles in eyes with high-pressure glaucoma group $(n=681$ eyes). Stroked line: tercile with high-pressure amplitude; dotted line: tercile with medium-pressure amplitude; thin line: tercile with low amplitude.

glaucoma subgroup ( $n=174$ eyes) with all IOP readings lower $22 \mathrm{mmHg}$ without medication, and the remaining high-pressure glaucoma subgroup ( $n=681$ eyes) revealed that, in the normal-pressure glaucoma group, progression was significantly associated with the minimal IOP value $(P<0.001)$, the mean of the IOP values $(P=0.024)$, and, negatively but less significantly $(P=0.037)$, with the profile amplitudes (Figure 1). In the high-pressure glaucoma group, the rate of progression was statistically independent of the IOP amplitude $(P=0.73)$ (Figure 2).

In the whole study population, the fluctuations of IOP in the pressure profiles were significantly $(P<0.001)$ and positively correlated with the minimal IOP $(P=-0.23)$, the maximal IOP $(P=0.59)$, and the mean IOP $(P=0.26)$. In a multiple Cox proportional hazards analysis, after forward selection, with the progression of glaucoma as dependent variable and age, neuroretinal rim area, the IOP amplitude, the minimal IOP value, the maximal IOP value, and the mean IOP values as independent variables, progression was significantly associated with age $(P<0.001)$ and neuroretinal rim area $(P=0.05)$ in the whole study population. In the high-pressure glaucoma subgroup only age $(P<0.001)$ was a significant prognostic factor, whereas in the normal-pressure glaucoma group, higher mean IOP $(P=0.036)$ and lower IOP amplitude $(P=0.045)$ were identified as independent significant predictors for glaucomatous progression.

\section{Discussion}

The result that the rate of progression of glaucoma was statistically not associated with the amplitude of intraocular measurements in the high-pressure glaucoma group confirms the recent Malmö Ocular Hypertension Study. ${ }^{12}$ In the latter, 90 patients were examined every 3 months with office-hours diurnal tension curves and computerised perimetry. After a maximum follow-up to 17 years, 37 patients had developed glaucomatous visual field defects. In univariate Cox regression analyses, mean IOP of all measurements was a significant risk factor for developing glaucoma, while IOP fluctuations were almost statistically significant. When separating effects of mean IOP level and mean IOP fluctuation, however, only the IOP level remained to be statistically significant. IOP fluctuations did not contribute to the risk, mainly since the pressure amplitude in the Malmö study as in the present investigation depended on the IOP level. Both studies are contradictory to a previous investigation by Asrani $e t a l^{5}$ who found that the diurnal IOP range and the IOP range over multiple days were significant risk factors for progression of glaucoma in 105 eyes of 64 patients. As in Asrani's study, univariate analysis in the present study suggested a significant association between the pressure profiles and the progression of normal-pressure glaucoma. Taking into account, however, the highly significant associations between the IOP amplitude and the mean, minimal and maximal IOP values suggests that it was the IOP itself, and less 
the pressure amplitude, which had the main influence on the rate of the glaucoma progression.

There are limitations of the present study. A major flaw in the design of this study was that the glaucoma eyes were receiving treatment by one or a combination of different topical IOP lowering medications. The effects of each of these medications on the IOP profile are largely unknown. Drug compliance may have further influenced the results. The resulting IOP profiles were thus the combined effects of a mix of treatment, compliance, and disease issues. Although it may suggest that the data cannot be taken to study the physiologic behaviour of the IOP in eyes with chronic open-angle glaucoma, the data of the present study may, however, reflect the daily clinical situation with patients with chronic open glaucoma under treatment. Another limitation of the study may be that since the IOP measurements depend on the corneal thickness ${ }^{16}$ and because corneal thickness may have decreased during daytime, the measurements may have been falsely low in the evening. All eyes included into the study, however, had clear corneas and a best corrected visual acuity of 20/25 or better. Another limitation of the study may be that the intraocular measurements at midnight were obtained in the sitting position at the slit lamp and not with the patients lying supine in bed. The information obtained in the present study can, therefore, only be transferred to the time between 0700 and midnight the time when many patients usually go to bed. Another limitation of the present study is, that the results may be valid only for eyes without preceding filtering surgery. In a recent study by Medeiros et al, ${ }^{17}$ the intraocular peak and fluctuation during diurnal pressure curve were significantly greater in a group of glaucoma patients with topical medical therapy than in a group of patients who had undergone filtering surgery. In an attempt to look for a further influence of IOP on the results of the study, the whole study population was divided into two subgroups based on the IOP without treatment. As cutoff point the value of $21 \mathrm{mmHg}$ was taken which has usually been the cutoff point for the definition of normal-pressure glaucoma. As another possible limitation of the study one may be aware that dividing the study population into the two subgroups may not imply that there is indeed a separate entity such as normal-pressure glaucoma.

Despite all these limitations of the study, one may conclude that the data suggest that the rate of glaucoma progression may be dependent more on the level of IOP than on the amount of IOP fluctuations in chronic open-angle glaucoma patients.

\section{References}

1 Heijl A, Leske MC, Bengtsson B, Hyman L, Bengtsson B, Hussein M, Early Manifest Glaucoma Trial Group. Reduction of intraocular pressure and glaucoma progression: results from the Early Manifest Glaucoma Trial. Arch Ophthalmol 2002; 120: 1268-1279.

2 Gordon MO, Beiser JA, Brandt JD, Heuer DK, Higginbotham EJ, Johnson CA et al. The Ocular Hypertension Treatment Study: baseline factors that predict the onset of primary open-angle glaucoma. Arch Ophthalmol 2002; 120: 714-720.

3 Jonas JB, Martus P, Horn FK, Jünemann AE, Korth M, Budde WM. Predictive factors of the optic nerve head for development or progression of glaucomatous visual field loss. Invest Ophthalmol Vis Sci 2004; 45: 2613-2618.

4 Konstas AG, Mantziris DA, Stewart WC. Diurnal intraocular pressure in untreated exfoliation and primary open-angle glaucoma. Arch Ophthalmol 1997; 115: 182-185.

5 Asrani S, Zeimer R, Wilensky J, Gieser D, Vitale S, Lindenmuth $\mathrm{K}$. Large diurnal fluctuations in intraocular pressure are an independent risk factor in patients with glaucoma. J Glaucoma 2000; 9: 134-142.

6 Wilensky JT, Gieser DK, Dietsche ML, Mori MT, Zeimer R. Individual variability in the diurnal intraocular pressure curve. Ophthalmology 1993; 100: 940-944.

7 Liu JHK, Zhang X, Kripke DF, Weinreb RN. Twenty-fourhour intraocular pressure pattern associated with early glaucomatous changes. Invest Ophthalmol Vis Sci 2003; 44: 1586-1590.

8 Liu JHK, Zhang X, Kripke DF, Weinreb RN. Nocturnal elevation of intraocular pressure is detectable in the sitting position. Invest Ophthalmol Vis Sci 2003; 44: 4439-4442.

9 Weinreb RN, Friedman DS, Fechtner RD, Cioffi GA, Coleman AL, Girkin CA et al. Risk assessment in the management of patients with ocular hypertension. Am J Ophthalmol 2004; 138: 458-467.

10 Mosaed S, Liu JHK, Weinreb RN. Correlation between office and peak nocturnal intraocular pressures in healthy subjects and glaucoma patients. Am J Ophthalmol 2005; 139: 320-324.

11 Weinreb RN. IOP and the risk of progression to glaucoma. Graefes Arch Clin Exp Ophthalmol 2005; 243: 511-512.

12 Bengtsson B, Heijl A. Diurnal IOP fluctuation: not an independent risk factor for glaucomatous visual field loss in high-risk ocular hypertension. Graefes Arch Clin Exp Ophthalmol 2005; 243: 513-518.

13 Jonas JB, Budde WM, Panda-Jonas S. Ophthalmoscopic evaluation of the optic nerve head. Surv Ophthalmol 1999; 43: 293-320.

14 Jonas JB, Martus P, Horn FK, Jünemann AE, Korth M, Budde WM. Predictive factors of the optic nerve head for development or progression of glaucomatous visual field loss. Invest Ophthalmol Vis Sci 2004; 45: 2613-2618.

15 Law CG, Brookmeyer R. Effects of mid-point imputation on the analysis of doubly censored data. Stat Med 1992; 11: 1569-1578.

16 Goldmann H, Schmidt T. ber Applanationstonometrie. Ophthalmologica 1957; 134: 221-222.

17 Medeiros FA, Pinheiro A, Moura FC, Leal BC, Susanna Jr R. Intraocular pressure fluctuations in medical versus surgically treated glaucomatous patients. Ocul Pharmacol Ther 2002; 18: 489-498. 